\title{
Efecto del riego con agua residual tratada sobre la calidad microbiológica del suelo y pasto King Grass
}

\section{Effect of irrigation with treated wastewater on microbiological quality of the soil and King Grass}

Harold E. González-Fragozoㅜㅜ Carolina Zabaleta-Solano²; Jessica Devia-González ${ }^{3}$; Yulieth Moya-Salinas ${ }^{4}$; Otilia Afanador-Rico ${ }^{5}$

\begin{abstract}
${ }^{1}$ Microbiólogo, Esp. Universidad Popular del Cesar, Grupo de Investigación Parasitología Agroecología Milenio. Valledupar - César, Colombia; e-mail: hegonzalez@unicesar.edu.co; (D) https://orcid.org/0000-0002-8219-0092

${ }^{2}$ Microbióloga. Universidad Popular del Cesar, Facultad Ciencias de la Salud. Valledupar - César, Colombia; e-mail: carolinazabaleta@hotmail.com; (D) https://orcid.org/0000-0002-1254-3155

${ }^{3}$ Microbióloga. Universidad Popular del Cesar, Facultad Ciencias de la Salud. Valledupar - César, Colombia; e-mail: jessica.devia29@gmail.com; (1D) https://orcid.org/0000-0001-7720-4413

${ }^{4}$ Microbióloga. Universidad Popular del Cesar, Facultad Ciencias de la Salud. Valledupar - César, Colombia; e-mail: juliethmoya1997@gmail.com; (D) https://orcid.org/0000-0003-0810-8617
\end{abstract}

${ }^{5}$ Microbióloga. Universidad Popular del Cesar, Facultad Ciencias de la Salud. Valledupar - César, Colombia; e-mail: otiafarico@gmail.com; (D) https://orcid.org/0000-0001-9051-2293

Cómo citar: González-Fragozo, H.E.; Zabaleta-Solano, C.; Devia-González, J.; Moya-Salinas, Y.; Afanador-Rico, O. 2020. Efecto del riego con agua residual tratada sobre la calidad microbiológica del suelo y pasto King grass. Rev. U.D.C.A Act. \& Div. Cient. 23(2):e1513. http://doi.org/10.31910/rudca.v23.n2.2020.1513

Artículo de acceso abierto publicado por Revista U.D.C.A Actualidad \& Divulgación Científica, bajo una licencia Creative Commons CC BY-NC 4.0

Publicación oficial de la Universidad de Ciencias Aplicadas y Ambientales U.D.C.A, Institución de Educación Superior Acreditada de Alta Calidad por el Ministerio de Educación Nacional.

Recibido: Abril 4 de 2020 Aceptado: Noviembre 24 de 2020 Editado por: Ingeborg Zenner de Polanía

\section{RESUMEN}

La contaminación del agua utilizada en el mundo y la falta de plantas de tratamiento altamente eficientes plantean problemas potenciales para la salud pública y el medio ambiente, por lo que los países buscan métodos confiables, inocuos y eficaces en función de los costos, para depurar las aguas residuales. Por otro lado, el sector agropecuario ha tomado auge, dada la necesidad de implementación de técnicas limpias, que sean amigables con el medio ambiente. El riego con aguas residuales en la agricultura, se ha vuelto común en regiones áridas y semiáridas, debido a la baja disponibilidad de agua, pero su uso requiere de un monitoreo cuidadoso de parámetros de higiene. El objetivo de este trabajo fue analizar el efecto del riego, con aguas residuales tratadas, sobre la contaminación microbiológica del suelo y el pasto King Grass, además de la producción de materia seca del forraje. Se evaluaron tres tratamientos: tratamiento T1: sin riesgo (SR), T2: agua subterránea (AS) y T3: agua residual tratada (ART). Se hicieron análisis fisicoquímicos y microbiológicos al agua, suelo y pasto. La contaminación microbiológica en el suelo regado con agua residual tratada no presentó diferencias significativas $(p>0,05)$ frente a los suelos donde se aplicó riego con agua subterránea, así como tampoco se presentaron diferencias estadísticas significativas entre variables microbiológicas en pasto. Se concluyó, que la fuente de contaminación microbiológica de 
los pastos y el suelo no está relacionada únicamente con la carga microbiana presente en las aguas residuales, sino que se puede deber, entre otros factores, a las escorrentías y al uso de aguas subterráneas contaminadas para el riego.

Palabras clave: Contaminación microbiológica; Materia seca; Biofertilización; Reutilización; Sostenibilidad.

\section{ABSTRACT}

World water contamination and the lack of wastewater treatment plants pose potential problems concerning public health and on the environment. Therefore, countries are looking for reliable, safe and cost-effective methods to purify wastewater. On the other hand, the agricultural sector has taken a lot of momentum given the need to implement clean techniques that are friendly to the environment. boomed given the need to implement clean techniques that are friendly to the environment. Wastewater irrigation in agriculture has become common in arid and semi-arid regions due to low water availability, but its use requires careful monitoring of hygiene parameters. The objective of this work was to analyze the effect of treated wastewater on microbiological contamination of the soil and King grass, in addition to the production of dry matter from forage. Were evaluated three treatments: T1: no risk; T2: groundwater and T3: treated wastewater. Were made physicochemical and microbiological analyzes out on water, soil and grass. The microbiological contamination in the soil irrigated with treated wastewater did not show significant differences compared to the soils where irrigation with groundwater was applied, as well as there were no statistical differences between microbiological variables in grass. It was concluded that the source of microbiological contamination of pastures and soil is not only related to the microbial load present in the wastewater, but it may be due, among other factors, to runoff and the use of contaminated groundwater for irrigation.

Keywords: Microbiological contamination; Dry matter; Biofertilization; Reuse; Sustainability.

\section{INTRODUCCIÓN}

El aumento constante de las aguas residuales producidas en zonas urbanas, industriales y comunitarias de todo el mundo plantea problemas potenciales para la salud pública y el ambiente (ONU, 2015). Debido a lo anterior, los países están buscando métodos seguros que sean sostenibles, confiables, inocuos y eficaces, en función de los costos, para depurar y eliminar las aguas residuales (Bonilla et al. 2013). Por otro lado, el sector agropecuario ha tomado mucho auge, dada la necesidad de implementación de técnicas limpias (Veliz et al. 2009) y que, además, los sistemas productivos sean sostenibles en el tiempo, sin ocasionar daños ambientales. El uso de aguas residuales para riego agrícola constituye una excelente oportunidad para proponer estrategias, que permitan combinar la disminución de las mismas, implicando técnicas de riego, mediante aspersión o goteo (Garzón et al. 2016), en cultivos que, preferiblemente, requieran procesamiento antes de su consumo (Veliz et al. 2009).

Las aguas residuales han sido usadas en la agricultura durante muchos años; en la Roma antigua, se utilizó como fertilizante (Pérez et al. 2019). En la actualidad, su uso ha aumentado, debido a la escasez de agua (Ghaitidak \& Yadav, 2013). Se aplican, principalmente, en cultivos que se procesan antes de su consumo, como maíz y arroz (Vera et al. 2016) y frutos que se consumen crudos; en menor medida, en algunos cultivos industriales, como algodón, además de árboles maderables y forrajes (Garzón et al. 2016).

Entre las especies forrajeras en las que se han usado aguas residuales para riego, se encuentran los pastos de corte (Fonseca et al. 2020), que se destacan por fácil adaptación a las condiciones de trópico seco (Martínez et al. 2020; Murillo et al. 2014). Una de las especies forrajeras más utilizadas en el caribe colombiano es el pasto King Grass, el cual, es un híbrido natural entre Pennisetum purpureum y $P$. typhoides (Martínez \& González, 2017). Este pasto fue ampliamente evaluado en la década de los 70 y parte de los 80 (Fonseca et al. 2020).

El King Grass es una gramínea perenne muy utilizada en fincas de regiones secas, como forraje para ganado bovino, ovino, caprino y porcino (Martínez \& González, 2017). Se adapta en zonas con alturas de 1.000 a $1.500 \mathrm{~m}$ s.n.m., con un rango amplio de distribución de lluvias y de fertilidad de suelos, incluyendo suelos ácidos de baja fertilidad (Ramos et al. 2015). Tiene un crecimiento erecto de sus macollos y alcanza alturas de hasta $5 \mathrm{~m}$ y sus tallos tienen un diámetro de 1,4 a 2,4cm (Kumar \& Goel, 2010); algunos reportes indican que el rendimiento se debe al manejo de las pasturas (Ramos et al. 2015).

Los sistemas productivos demandan grandes cantidades de agua, la cual, se hace cada vez más escaza (Martínez \& González, 2017), por ello, se han implementado estrategias de riego de forrajes con aguas residuales. Esta práctica, se ha vuelto común en regiones áridas y semiáridas, debido a la baja disponibilidad de agua, pero su uso requiere de un monitoreo cuidadoso de parámetros de higiene (FAO, 2013b). De los países que riegan con aguas residuales tratadas, sobresalen China, Colombia y México, con 1.300.000, 1.230.193 y 360.000 hectáreas, respectivamente (FAO, 2017). También, se destacan Vietnam, donde el $80 \%$ de sus hortalizas se irriga con agua residual tratada, mezclada con agua potable y Ghana, con 2.900 hectáreas de especies forrajeras, regadas con aguas servidas sin tratar (FAO, 2013a).

La falta de agua es un fenómeno presente en regiones de todo el mundo. El Departamento del Cesar no es ajeno a esta problemática, puesto que es uno de los departamentos colombianos con menor disponibilidad de agua para riego (uso agrícola), aspecto al que se le atribuye una alta responsabilidad sobre la baja producción agrícola regional (IDEAM, 2017), lo hace evidente la necesidad de buscar nuevas fuentes hídricas, considerándose a las aguas residuales tratadas una fuente adicional, las cuales, además, están provistas de un alto contenido de nutrimentos aprovechables por la planta $(\mathrm{Lu}$ et al. 2016). 
El objetivo de este trabajo fue analizar el efecto del riego, con aguas residuales tratadas, sobre la contaminación microbiológica del suelo y el pasto King Grass, además de la producción de materia seca del forraje.

\section{MATERIALES Y MÉTODOS}

La presente investigación, se realizó en el municipio de Agustín Codazzi del departamento del Cesar, que presenta promedio de temperaturas anuales de $29,5^{\circ} \mathrm{C}$ y precipitaciones de $1.501 \mathrm{~mm}$ (IDEAM, 2020). La fase experimental, se desarrolló entre mayo y diciembre de 2019, en la finca Santa Rita, ubicada a los 1002'37,1" latitud Norte (N) y 73¹3'01,3"' longitud Oeste (W); el área de estudio cuenta con una extensión de 812 ha a $131 \mathrm{~m}$ s.n.m.

Diseño experimental. Para el experimento, se establecieron las plantas King Grass (Pennisetum prupureum y P. typhoides). Se utilizó el método de gradiente en riego por aspersión semiestacionario, con diseño en las parcelas y tratamientos no aleatorios. El área total de experimento fue de $0,66 \mathrm{ha}$, distribuido en 9 parcelas de $600 \mathrm{~m}^{2}$ (20x30m), cada una con espacio de $4 \mathrm{~m}$ entre ellas; en cada parcela, se establecieron 10 surcos, con 1,5m de distancia, se sembraron 12 esquejes de pasto, en cada surco.

Se evaluaron tres tipos de tratamientos con tres repeticiones cada uno: Tratamiento 1 (T1), sin riego especial - condiciones de secano (SR); Tratamiento 2 (T2), riego con agua subterránea (AS) y Tratamiento 3 (T3), riego con agua residual tratada (ART). En las parcelas de pasto King Grass establecidas en los tratamientos 2 y 3 y sus repeticiones; se emplearon 6 aspersores, con alcance de $10 \mathrm{~m}$ cada uno. El agua subterránea utilizada en el riego de los pastos, se obtuvo de los pozos de la finca Santa Rita, mientras que el agua residual tratada, por la Planta de Tratamiento de Aguas Residuales del municipio de Agustín Codazzi - Cesar, cuyo tratamiento se realiza mediante lagunas de oxidación.

Análisis del agua residual. Se realizó determinación de Coliformes Totales (CT) y Fecales (CF), por la técnica NMP; DBO, por el test DBO 5 días; DQO, por el método de reflujo abierto; concentración de nitratos, por el método de reducción de cadmio; fósforo total, por el método del ácido ascórbico; solidos suspendidos totales, por gravimetría y turbiedad, por nefelometría. Los procedimientos, se realizaron teniendo en cuenta los métodos estándares de la American Water Works Association (AWWA, 2017).

Análisis fisicoquímico del suelo. Se tomaron muestras de suelo siguiendo el protocolo propuesto por el Instituto Geográfico Agustín Codazzi (2007). Las propiedades fisicoquímicas, se analizaron solo en los tratamientos regados con ART, para lo cual, se tomaron muestras de suelo antes de la preparación para la siembra y después del último riego con ART; se analizó textura, humedad, capacidad de campo, densidad aparente, porosidad y capacidad de infiltración. También los parámetros químicos: pH, concentración de Materia Orgánica, Cloruros, Sulfatos, Nitratos, $\mathrm{DBO}_{5}$ (Demanda Bioquímica de Oxígeno), Potasio, Fósforo, Zinc, Nitrógeno total y Cobre.
Las técnicas de laboratorio para determinación de características fisicoquímicas del suelo, se realizaron teniendo en cuenta los métodos estándares de la American Water Works Association (AWWA, 2017).

Análisis microbiológico del suelo y pastos. Se tomaron muestras aleatorias de $500 \mathrm{~g}$ de suelo, a $10 \mathrm{~cm}$ de profundidad antes y una vez finalizado el riego. De cada muestra, se tomó una alícuota de $25 \mathrm{~g}$ para la cuantificación de Coliformes Totales y Fecales, mediante la técnica de NMP (APHA, 1992). Para el caso de los pastos, se realizó recuento de CT y Escherichia coli, por recuento en placa, en medio Chromocult ${ }^{\circledR}$ Coliform Agar; detección de Pseudomonas aeruginosa, por siembra en agar cetrimide y Pithomyces chartarum, por siembra en PDA y agar-agar enriquecido, con jugo V8; claves taxonómicas y las descripciones de Canafoglia et al. (2007).

Determinación del contenido de materia seca del pasto King Grass. Para estimar los valores de nutrición y de rentabilidad de los pastos, una vez finalizado el experimento, se determinó el contenido de materia seca (MS) (Ramos et al. 2015). Para ello, se tomaron tres muestras en dos surcos centrales, descartando los bordes de cada parcela; a una submuestra $(250 \mathrm{~g})$, se le determinó peso seco, según metodología de 't Mannetje \& Jones (2000) y con base en ello, se calculó el porcentaje de MS.

Análisis estadístico. Se realizó Análisis de Varianza de un factor para comparar las medias correspondientes a cada tratamiento de las variables peso seco, materia seca y coliformes totales en pasto; también, se realizó el mismo análisis para las variables coliformes totales y coliformes fecales en muestras de suelo, de cada uno de los tratamientos evaluados. En aquellas variables en las que el valor de la probabilidad fue inferior a $0,05(\mathrm{p}<0,05)$, se realizó la prueba de Tukey, para comparar las medias de los tratamientos en función de la magnitud de los valores obtenidos. En análisis estadístico, se realizó con el software Statistical Package for the Social Sciences (SPSS) versión 23.

\section{RESULTADOS Y DISCUSIÓN}

Análisis agua residual. El ART utilizada en esta investigación tuvo una concentración de $1,6 \times 10^{3}$ de CT y $0,9 \times 10^{2}$ de CF, por lo tanto, se consideró viable al cumplir con los criterios microbiológicos establecidos en la Resolución 1207 de 2014 (MinAmbiente, 2014) cuyos valores máximos permisibles son de $1,0 \mathrm{X} 10^{5}$ para CT y $1,0 \mathrm{X} 10^{2}$ para CF (Tabla 1). En cuanto a los parámetros fisicoquímicos, el ART tuvo $\mathrm{pH}$ de 7,4, turbiedad de 6 NTU, sólidos suspendidos totales de $123 \mathrm{mg} / \mathrm{L}$, los niveles de Cloruro, Sulfatos y Nitratos fueron de $14 \mathrm{mg} / \mathrm{L}, 329 \mathrm{mg} / \mathrm{L}$ y $16 \mathrm{mg} / \mathrm{L}$, respectivamente y la DBO fue de $28,4 \mathrm{mg} / \mathrm{L}$ (Tabla 1). El ART cumple con los criterios fisicoquímicos de la Resolución 1207 de 2014 (MinAmbiente, 2014) y con las directrices impartidas por la Agencia de Protección Ambiental para el reúso de aguas residuales (EPA, 2012), para ser utilizada con fines agrícolas, a excepción de los nitratos, los cuales, se calcularon por encima del valor mínimo permisible, de acuerdo con la norma. 
Tabla 1. Análisis del agua residual.

\begin{tabular}{|c|c|c|c|}
\hline Variable & Resultado & $\begin{array}{l}\text { Valor Límite Máximo Permisible } \\
\text { Resolución } 1207 \text { de } 2014 \\
\text { (MinAmbiente, 2014) }\end{array}$ & $\begin{array}{l}\text { Unidad de } \\
\text { Medida }\end{array}$ \\
\hline Coliformes totales & $1600 \times 10^{2}$ & $1,0 \times 10^{-5}$ & \multirow{2}{*}{$\mathrm{NMP} / 100 \mathrm{ml}$} \\
\hline Coliformes fecales & $0,9 \times 10^{2}$ & $1,0 \times 10^{-2}$ & \\
\hline $\mathrm{DBO}_{5}$ & 28,4 & 30 & $\mathrm{mg} \mathrm{O}_{2} / \mathrm{L}$ \\
\hline Nitratos & 16 & 5 & $\mathrm{NO}_{3} / \mathrm{L}$ \\
\hline Sulfatos & 329 & 500 & $\mathrm{mg} \mathrm{SO}{ }_{4}^{2-} / \mathrm{L}$ \\
\hline Cloruros & 141 & 300 & $\mathrm{Mg} \mathrm{Cl}^{-} / \mathrm{L}$ \\
\hline Solidos suspendidos totales & 123 & - & $\mathrm{mg} / \mathrm{L}$ \\
\hline Turbiedad & 6 & - & NTU \\
\hline $\mathrm{pH}$ & 7,4 & $6,0-9,0$ & Unidades de $\mathrm{pH}$ \\
\hline
\end{tabular}

En Colombia, se han implementado estrategias para reutilizar el agua residual tratada en la agricultura, bajo el cumplimiento estricto de parámetros y criterios establecidos por el Ministerio de Ambiente y Desarrollo Sostenible; sin embargo, hay estudios en donde se utilizan ART que presenta valores superiores en uno o más parámetros, tal como es el caso de esta investigación, con los valores de Nitratos, que se reportaron por encima de los mínimos reglamentados, al igual que al ART, utilizada por Hernández et al. (2014), para cultivos forrajeros de riego, cuyos valores de parámetros químico presentaron rangos superiores a los permitidos por la normativa colombiana.

Tabla 2. Análisis fisicoquímico del suelo.
Análisis fisicoquímico del suelo. En la tabla 2, se observa que los parámetros Fósforo $(\mathrm{P})$, Potasio $(\mathrm{K})$ y Materia Orgánica (MO) aumentaron ligeramente, debido a que el clima tiene mayor influencia sobre estas características, lo que concuerda con lo reportado por Hernández et al. (2014), quienes señalaron que en suelos con riego intermitente de aguas servidas no hay incremento significativo de los niveles de $\mathrm{P}, \mathrm{K}$, y MO. En adición a esto, Wu \& Chang (2018) publicaron que con el Zinc ocurre lo contrario, es decir, se aumenta su contenido, dadas las elevadas cantidades de este elemento presente en las aguas residuales.

\begin{tabular}{|c|c|c|}
\hline PARÁMETROS & ANTES DEL ENSAYO & DESPUÉS DEL ENSAYO \\
\hline \multicolumn{3}{|c|}{ Físicos } \\
\hline Textura & Franco arenoso & Franco arenoso \\
\hline Humedad $(\mathrm{H})$ & $1,53 \%$ & $2,12 \%$ \\
\hline Capacidad de campo & $27,78 \%$ & $27,81 \%$ \\
\hline Velocidad de filtración & $0,34 \mathrm{~m} / \mathrm{s}$ & $0,42 \mathrm{~m} / \mathrm{s}$ \\
\hline Densidad aparente & $0,76 \mathrm{~g} / \mathrm{cm} 3$ & $0,81 \mathrm{~g} / \mathrm{cm} 3$ \\
\hline Porosidad & $65,9 \%$ & $69,23 \%$ \\
\hline \multicolumn{3}{|c|}{ Químicos } \\
\hline $\mathrm{pH}$ & 6,23 & 6,42 \\
\hline Materia orgánica & $116,98 \mathrm{~g} / \mathrm{kg}$ & $117,45 \mathrm{~g} / \mathrm{kg}$ \\
\hline Fósforo $(\mathrm{P})$ & $0,22 \mathrm{mg} / \mathrm{kg}$ & $0,69 \mathrm{mg} / \mathrm{kg}$ \\
\hline Nitrógeno total & $0,06 \% \mathrm{~m} / \mathrm{m}$ & $0,67 \% \mathrm{~m} / \mathrm{m}$ \\
\hline Potasio $(\mathrm{K})$ & $428 \mathrm{mg} / \mathrm{kg}$ & $433 \mathrm{mg} / \mathrm{kg}$ \\
\hline Zinc $(\mathrm{Zn})$ & $1,21 \mathrm{mg} / \mathrm{kg}$ & $1,37 \mathrm{mg} / \mathrm{kg}$ \\
\hline Cobre $(\mathrm{Cu})$ & $0,14 \mathrm{mg} / \mathrm{kg}$ & $0,19 \mathrm{mg} / \mathrm{kg}$ \\
\hline
\end{tabular}


Contrario a lo anterior, se observa un aumento del Nitrógeno Total de $0,06 \%$ a $0,67 \%$. Estos porcentajes han sido reportados por Kumar \& Goel (2010), dentro de los valores promedio, en suelos ricos en Nitrógeno (N). Con relación a los cambios en la concentración del $\mathrm{N}$ en el suelo, Tolentino et al. (2019) demostraron el potencial de las aguas residuales en la fertilización del sustrato, además de estar relacionado con la alta concentración de este elemento en el agua usada (Tartabull \& Betancourt, 2016). Aunque hubo un aumento de Cobre en el suelo, la concentración de este elemento no representa riesgo de contaminación, puesto que la concentración determinada en este estudio, no supera el límite máximo de toxicidad, el cual, es de 30mg/kg (Resolución 1207 de 2014) (MinAmbiente, 2014).

Análisis microbiológico del suelo. Se determinó presencia de CT $\mathrm{T}$ y CF en el suelo de todos los tratamientos antes y después del riego (Figura 1), lo que indica que el riego con agua residual tratada no es la única fuente de contaminación microbiológica del suelo (Santacoloma et al. 2020).

En la Figura 1a, se aprecia el resultado del recuento de CT en muestras de suelo bajo los tres tratamientos; en el tratamiento SR, el recuento de CT antes del riego fue de $1,3 \times 10^{4}$. Los valores de CT después del riego fueron ligeramente superiores $\left(13,5 \times 10^{3}\right)$, por lo que no hubo diferencias significativas entre ambos valores $(p=0,053)$. Al igual que los coliformes totales, el comportamiento de los CF se mantuvo estable, puesto que se reportaron recuento inicial (antes del riego) y final (después del riego), de $2,53 \times 10^{3}$ y $2,62 \times 10^{3}$, respectivamente (Figura 1b). Es importante mencionar que los pastos sembrados en suelos de las parcelas del tratamiento SR y sus repeticiones no fueron regados, se mantuvieron en condiciones de
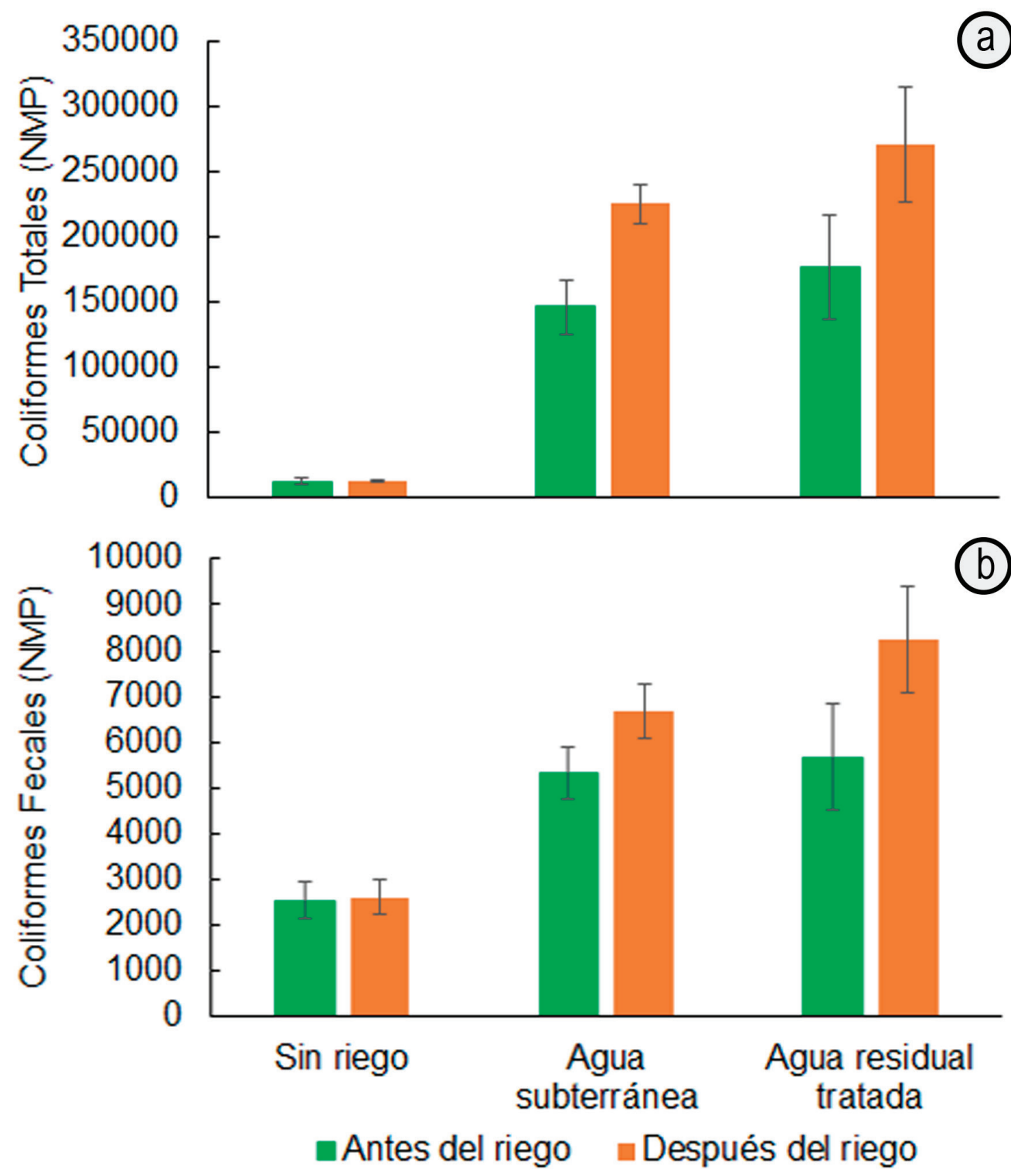

Figura 1. Cuantificación de coliformes en suelo (NMP/100g). 
secano (Vera \& Hoyos, 2019), recibiendo irrigación únicamente de las precipitaciones, que hubo durante el tiempo de estudio.

Los valores finales del recuento de CT y CF aumentaron en los tratamientos regados con AS y ART, lo que indica un aumento estadísticamente importante de la concentración de dichos indicadores de contaminación microbiológica; sin embargo, en los suelos de los tratamientos AS se determinó presencia de CT y CF, población bacteriana que aumentó después del riego con agua subterránea, presentando diferencias significativas con los valores de recuento inicial $(\mathrm{p}=0,02)$. Por lo tanto, se asume que la contaminación por coliformes en el suelo no es generada por el riego con aguas residuales, sino por fuentes distintas a ésta.

Lo anterior supone un punto a favor para el uso de ART en la agricultura, aunque la implementación de aguas usadas para el riego agrícola podría aumentar la concentración de coliformes (Santacoloma et al. 2020), tal y como se evidencia en las figuras 1a y $1 \mathrm{~b}$, donde se presentaron diferencias significativas $(\mathrm{p}=0,01)$ entre los recuentos de CT antes y después del riego con ART, cuyos valores fueron de $17,6 \times 10^{4}$ y $27,1 \times 10^{4}$, así como también se reporta aumento en recuento de CF de $5,6 \times 10^{3}$ hasta $8,2 \times 10^{3}$, los cuales, difieren estadísticamente entre sí. Estudios realizados por Vera et al. (2016) reportaron altas concentraciones de CT, CF y E. coli en suelos regados con agua subterránea y aguas residuales, respectivamente. Al ser comparados estas observaciones con los actuales resultados, se deduce que la contaminación del suelo al final del experimento fue baja, lo que coincide con lo que encontraron Chuquimboques et al. (2019), quienes atribuyeron la contaminación a la frecuencia e intensidad de riego, lo que indica, que el tiempo en que se aplique el riego con agua residual tratada podría influir también en la contaminación del suelo.

Análisis microbiológico del pasto. El pasto establecido en los tratamientos SR presentó 3,5X10 ${ }^{2} \mathrm{UFC} / \mathrm{g}$ de $\mathrm{CT}$, valores que presentaron diferencias estadísticas con los recuentos de CT en pastos obtenidos de los tratamientos AS y ART, cuyos valores de $\mathrm{UFC} / \mathrm{g}$ correspondieron a $3,7 \times 10^{3}$ y $5,6 \times 10^{3}$, respectivamente (Tabla 3).

Tabla 3. Recuentos de coliformes totales y E. coli en el pasto (UFC/g) y contenido de materia y peso seco.

\begin{tabular}{|c|c|c|c|c|}
\hline Tratamiento & Coliformes Totales \pm DS & E. coli & $\begin{array}{c}\text { Peso Seco } \pm \text { Ds } \\
(\mathbf{g})\end{array}$ & Materia Seca \pm Ds (\%) \\
\hline SR & $3,5 \times 10^{2} \pm 1,8 \times 10^{2} \mathrm{~b}$ & $<10$ & $35,67 \pm 2,38 \mathrm{c}$ & $14,27 \pm 0,96 \mathrm{c}$ \\
\hline AS & $3,7 \times 10^{3} \pm 1,4 \times 10^{3} \mathrm{~b}$ & $<10$ & $57,50 \pm 2,05 \mathrm{~b}$ & $23,00 \pm 0,82 \mathrm{~b}$ \\
\hline ART & $5,6 \times 10^{3} \pm 1,1 \times 10^{3} \mathrm{a}$ & $<10$ & $69,83 \pm 2,43 \mathrm{a}$ & $27,93 \pm 0,97 \mathrm{a}$ \\
\hline
\end{tabular}

Medias con diferente letra son significativamente diferentes según la prueba de Tukey

La presencia de CT en pastos crecidos en el tratamiento SR, se puede deber a distintos factores, como lo son la contaminación de los esquejes o la carga bacteriana presente en el suelo antes y durante la siembra, sin embargo, la contaminación con CT de los pastos puede tener orígenes en el arrastre, ocasionado por las corrientes de agua de lluvia durante el experimento, dado que, la elevación de tierra permitió el arrastre de material desde el T3, pasando por el T2 hasta el T1, a través de escorrentías de agua.

La concentración de coliformes cuantificados en los pastos regados con AS no presentó diferencias significativas $(\mathrm{p}=0,06)$ con los datos obtenidos de los pastos regados con ART, en cuanto al grado de contaminación (Tabla 3); sin embargo, al contrastar los datos obtenidos en los tratamientos mencionados frente a resultados del recuento de coliformes en pastos del tratamiento SR, se observa una diferencia significativa $(p=0,03)$, de acuerdo con la prueba de Tukey.

Estudios sobre la calidad microbiológica de alimentos de consumo crudo y pienso de animales han sido realizados en diversos cultivos. Al respecto, Lasso \& Ramírez (2011) reportaron varias fuentes de contaminación de estos alimentos, dentro de ellos, se encuentra el contacto de heces de animales silvestres con los alimentos. Por otro lado, Montero et al. (2016) atribuyeron la presencia de coliformes a la contaminación cruzada por las corrientes que se forman con aguas de lluvia, las cuales, arrastran y transportan varios componentes de la capa más superficial del suelo.

Es válido resaltar que en ninguno de los tratamientos se detectó la presencia de E. coli, lo que concuerda con lo señalado por Özlem \& Sener (2015), quienes reportaron ausencia de E. coli en plantas de maíz irrigadas con agua subterránea y agua residual agroindustrial tratada. La ausencia de este indicador universal, se puede deber a la alta radiación solar a la que estuvieron expuestas las hojas durante el ensayo. Por otra parte, la frecuencia e intensidad de riego contribuyeron a reducir el efecto de las aguas residuales sobre la microfauna.

Adicional a la ausencia de E. coli, se reporta la ausencia de Pseudomonas aeruginosa y $P$. chartarum. Actualmente, no se han reportado infecciones por $P$. aeruginosa en pasto King Grass y mientras que $P$. chartarum, se asocia a infecciones en pasto de la especie Brachiaria brizantha, debido a su alta especificidad (Libutti et al. 2018). 
Contenido de materia seca del pasto King Grass. El mayor contenido de materia seca, se obtuvo en el tratamiento de las plantas regadas con ART (Tabla 3), lo que concuerda con los reportes de Wang et al. (2019) y Bryan \& Irving (1987), quienes obtuvieron mejores rendimientos de plantas de maíz, cuando fueron regadas con aguas residuales domésticas. Además, los autores atribuyen el aumento en el peso seco de las plantas al alto contenido de nutrientes característico de las aguas residuales, los cuales, directamente aumentan la productividad de los cultivos (Hernández et al. 2014).

El agua es un elemento fundamental para las funciones vitales de los seres vivos, además, participa en los procesos metabólicos intracelulares; por ello, los pastos que fueron regados con AS presentaron mayores porcentajes de peso seco que los pastos que no fueron regados. Por otra parte, el porcentaje de Materia Seca del pasto SR es similar a los reportados por Wang et al. (2019), en Pennisetum purpureum, durante épocas de sequía, debido a la resistencia al estrés hídrico (Lasso \& Ramírez, 2011).

El mayor contenido de materia seca, se obtuvo en el pasto regado con ART (27,93\%), debido a la cantidad de nutrimentos que posee este tipo de aguas (Montero et al. 2016), seguido del pasto regado con AS, con 23\% y, por último, el pasto sin riego, con 14\% (Tabla 3). La diferencia significativa que existe entre los tres tratamientos $(p<0,05)$ permite asociar el aumento de la materia seca y rendimiento del pasto al riego con aguas subterráneas y aguas residuales tratadas. $\mathrm{Al}$ respecto se menciona que las ART tienen alto valor fertilizante, debido a los niveles de N, P y K y que contribuyen al desarrollo y formación de tejidos en las plantas (Andrade et al. 2017).

El uso de ART para riego agrícola es una estrategia amigable con el medio ambiente, que aumenta la humedad, la velocidad de filtración y la porosidad en los suelos y genera diversos beneficios a los cultivos de pastos para consumo animal, debido al contenido de macro y micronutrientes, que le confieren un carácter fertilizante, que mejoran el rendimiento de los pastos, en cuando al peso seco y contenido de materia seca.

La presencia de Coliformes en suelo y pastos, cuando se aplica riego con aguas residuales, no se debe únicamente a la carga microbiana del agua reutilizada, pues estos microorganismos llegan al suelo por distintas fuentes, por lo tanto, la contaminación microbiana de suelos y pastos no se debe al uso de aguas residuales tratadas en el riego.

Conflicto de intereses: El artículo fue preparado y revisado con la participación de todos los autores, quienes declaramos que no existe conflicto de intereses que ponga en riesgo la validez de los resultados presentados en el documento.

\section{REFERENCIAS}

1. AMERICAN WATER WORKS ASSOCIATION, AWWA. 2017. Standard Methods for the examination of water and wastewater. Ed. HARDBACK (EEUU). 84p.
2. ANDRADE, J.; BEZERRA, I.; SOUSA, O.; SILVA, N.; CHIPANA, R.; CRUZ, J.; DUNGA, J. 2017. Contenido foliar de N, P y K en algodón fertirrigado con efluente de alcantarillado tratado. DYNA. 84(202):147-154. https://dx.doi.org/10.15446/dyna.v84n202.61916

3. APHA. 1992. APHA Method 9221: Standard Methods for the Examination of Water and Wastewater. 18th ed. Disponible desde Internet en: https://law.resource.org/pub/us/cfr/ibr/002/apha. method.9221.1992.pdf (con acceso 18/07/2020).

4. BONILLA, M.; SILVA, S.; CABRERA, M.; SÁNCHEZ, T. 2013. Calidad del agua residual no entubada vertida por dos parques industriales en la ciudad de Puebla, México. Rev. Iberoam. Investig. Des. Ed. 4(7):1-36

5. BRYAN, K.; IRVING, L. 1987. Virus survival on vegetables spray-irrigated with wastewater. Water Research. 21(81):5763. https://doi.org/10.1016/0043-1354(87)90099-6

6. CANAFOGLIA, M.; COMERIO, R.; FERNÁNDEZ, V.; VAAMONDE, G. 2007. Hongos toxicogénicos contaminantes de frutos de alpataco. Rev. Iberoamericana de Micología. 24(1):56-58. https://doi.org/10.1016/S1130-1406(07)70014-1

7. CHUQUIMBOQUES, J.; VERGARA, J.; MENDOZA, J. 2019. Optimización de la remoción simultánea de nitrato, nitrito, amonio y fosfato de aguas residuales municipales. Rev. Soc. Química del Perú. 85(1):85-96.

8. EPA. 2012. Guidelines for Water Reuse. Disponible desde Internet en:

http://nepis.epa.gov/Adobe/PDF/P100FS7K.pdf (con acceso 14/02/2020).

9. FAO. 2013a. El contexto mundial. En: Reutilización del agua en la agricultura: ¿Beneficios para todos? Disponible desde Internet en:

http://www.fao.org/docrep/017/i1629s/i1629s.pdf (con acceso 20/07/2020).

10. FAO. 2013b. Reciclaje y reutilización de agua. En: Afrontar la escasez de agua. Un marco de acción para la agricultura y la seguridad alimentaria. (Informe sobre temas hídricos). Disponible desde Internet en: http://www.fao.org/docrep/018/i3015s/i3015s.pdf (con acceso $12 / 07 / 2020)$.

11. FAO. 2017. Reutilización de aguas para agricultura en America Látina y el Caribe. Estado, principios y necesidades. Organización de las naciones unidas para la alimentación 
y la agricultura (FAO). Mateo-Sagastra, J. (ed.). Disponible desde Internet en:

http://www.fao.org/3/a-i7748s.pdf (con acceso 17/07/2020).

12. FONSECA, D.; BOHÓRQUEZ, I.; RODRÍGUEZ, C.; VIVAS, N. 2020. Efecto del periodo de recuperación en la producción y calidad nutricional de algunas especies forrajeras. Biotecnología en el Sector Agropecuario y Agroindustrial. 18(2):135-144.

https://dx.doi.org/10.18684/bsaa(18)135-144

13. GARZÓN, Z.; GONZÁLEZ,Z.; GARCÍA, B. 2016. Evaluación de un sistema de tratamiento doméstico para reúso de agua residual. Rev. Internal Contaminación Ambiental. 32(2):199-211.

https://doi.org/10.20937/RICA.2016.32.02.06

14. GHAITIDAK, D.; YADAV, K. 2013. Characteristics and treatment of greywater- a review. Environ. Sci. Pollut. Res. 20(5):2795-2809.

https://doi.org/10.1007/s11356-013-1533-0

15. HERNÁNDEZ, E.; QUIÑONES, E.; CRISTOBAL, D.; RUBIÑOS, J. 2014. Calidad biológica de aguas residuales utilizadas para riego de cultivos forrajeros en Tulancingo, Hidalgo, México. Rev. Chapingo, Ser. Cienc. Forest. Amb. 1(20):89-100.

https://doi.org/10.5154/r.rchscfa.2012.03.024

16. IDEAM. 2017. Estimación de la demanda del agua. En: Estudio Nacional del Agua ( $5^{\circ}$ informe). Disponible desde Internet en:

http://www.siac.gov.co/documentos/DOC_Portal/ DOC_Agua/4_Presion/20120928_Estim_deman_agua_ ENA_2010.pdf (con acceso 17/07/2020).

17. IDEAM. 2020. Registros climatológicos y limninográficos, estación Valledupar. Disponible desde internet en: http://www.ideam.gov.co/solicitud-de-informacion (con acceso 10/08/2020).

18. INSTITUTO GEOGRÁFICO AGUSTÍN CODAZZI. 2007. Manual de métodos analíticos. Instructivo para toma de muestras de suelo. Instituto Geográfico Agustín Codazzi (Bogotá). p.4-5. Disponible desde internet en: https://www.igac.gov.co/sites/igac.gov.co/files/ guiademuestreo.pdf (con acceso 21/07/2020).

19. KUMAR, N.; GOEL, S. 2010. Factors influencing arsenic and nitrate removal from drinking water in a continuous flow electrocoagulation (EC) process. J. Hazardous Materials. 173(1-3):528-533. https://doi.org/10.1016/j.jhazmat.2009.08.117
20. LASSO, J.; RAMIREZ, J. 2011. Perspectivas generales del efecto del reúso de aguas residuales para riego en cultivos para la producción de biocombustibles en Colombia. El hombre y la máquina. 36:95-105.

21. LIBUT'TI, A.; GATTA, G.; GAGLIARDI, A.; VERGINE, P.; POLLICE, A.; BENEDUCE, L.; DISCIGLIO, G.; TARANTINO, E. 2018. Agro-industrial wastewater reuse for irrigation of a vegetable crop succession under Mediterranean conditions. Agricultural Water Management. 196:1-14. https://doi.org/10.1016/j.agwat.2017.10.015

22. LU, Y.; ZHAO, C.; MENG, Z.; WANG, X.; WU, G.; GAO, N. 2016. Straw friction characteristic based on rotary cutting anti-blocking device with slide plate pressing straw. Transactions of the Chinese Society of Agricultural Engineering. 17(11):83-89.

23. MARTÍNEZ, C.; VIVAS, N.; MORALES, S. 2020. Agronomic response of forage mixtures in a silvopastoral system in the Colombian dry tropics. DYNA. 87(213):80-84. https://dx.doi.org/10.15446/dyna.v87n213.79900

24. MARTÍNEZ, R.; GONZÁLEZ, C. 2017. Evaluation of varieties and hybrids of elephant grass Pennisetum purpureum and Pennisetum purpureum x Pennisetum glaucum for forage production. Cuban J. Agricultural Science. 51(4):477-488.

25. MINAMBIENTE MINISTERIO DE AMBIENTE Y DESARROLLO SOSTENIBLE. 2014. Resolución 1207 DE 2014 (julio 25) Diario Oficial No. 49.242 de 13 de agosto de 2014. Por la cual se adoptan disposiciones relacionadas con el uso de aguas residuales tratadas. Disponible desde Internet en:

https://www.icbf.gov.co/.../docs/resolucion_ minambienteds_1207_2014.htm (con acceso 15/08/2019)

26. MONTERO, S.; NIKOLSKII, I.; LANDEROS, C.; PALACIOS, O.; TRAVERSONI, L.; HERNÁNDEZ, J. 2016. Understanding the vegetable contamination process with parasites from wastewater irrigation and its impact on human health in Hidalgo, Mexico. J. Agric. Sc. 5(8):42-49. https://doi.org/10.5539/jas.v8n5p42

27. MURILlO, J.; BARROS, J.; RONCALlO, B.; ARRIETA, G. 2014. Requerimientos hídricos de cuatro gramineas de corte para uso eficiente del agua en el caribe seco colombiano. Corpoica Cienc. Tecnol. Agropecu. 15(1):83-99. https://doi.org/10.21930/rcta.vol15_num1_art:399

28. ONU. 2015. Organización de las Naciones Unidas. Informe de las naciones unidas sobre los recursos hídricos en el mundo: Agua para un mundo sostenible. Resumen ejecutivo. 06134 Colombella, Perugia, Italia. p.1-8. Disponible desde internet en: 
http://www.unesco.org/new/es/naturalsciences/ environment/water/wwap/wwdr/2015-water-for-asustainable-world/ (con acceso 19/07/2020).

29. ÖZLEM, E.; SENER, H. 2015. The contamination of various fruit and vegetable with Enterobius vermicularis, Ascaris eggs, Entamoeba histolytica cyst and Giardia cysts. Food Control. 16:559-562.

https://doi.org/10.1016/j.foodcont.2004.06.016

30. PÉREZ, J.; ORTEGA, H.; RAMÍREZ, C.; SÁNCHEZ, E.; CAN-CHULIM, Á.; MANCILLA, O. 2019. Evaluación de la calidad del agua residual para el riego agrícola en Valle del Mezquital, Hidalgo. Acta universitaria. 42(2):89-102. https://doi.org/10.15174/au.2019.2117

31. RAMOS, O.; VICTORIA, C.; SANDOVAL, J. 2015. Season, fertilization, and yield of varieties of Pennisetum purpureum. Agrociencia. 49(8):837-844.

32. SANTACOLOMA, S.; BUITRAGO, M.; COLORADO, K.; SUÁREZ, I.; MARTÍNEZ, M.; VILLEGAS, L. 2020. Agricultural use of biosolids generated in wastewater treatment of a food industry. Rev. Facultad Ingeniería. 29(54):106-121.

https://dx.doi.org/10.19053/01211129.v29. n54.2020.10666

33. T MANNETJE, L.; JONES, R.M. 2000. Grassland vegetation and its measurement. En: 't Mannetje, L.; Jones, R.M. (Eds). Field and laboratory methods for grassland and animal production research. CABI e-book. p.1-8. https://doi.org/10.1079/9780851993515.0103

34. TARTABULL, P.; BETANCOURT, A. 2016. La calidad del agua para el riego. Principales indicadores de medida $\mathrm{y}$ procesos que la impactan. Rev. Científ. Agroecos. 4(1):4761.
35. TOLENTINO, A.; BENITES, E.; CABRERA, C. 2019. Aguas residuales de lavandería y su tratamiento por Oxidación fotocatalítica con dióxido de titanio (TiO2) y luz ultra violeta (UV) en Instituto Nacional de Salud del Niño, San Borja- 2017. Rev. Instituto Investig. Fac. Ingeniería Geológica, Minera, Metalúrgica Y Geográfica. 22(43):3-8.

36. VELIZ, L.; LLANES, O.; ASELA, F.; BATALLER, M. 2009. Reúso de aguas residuales domésticas para riego agrícola. Valoración crítica. Rev. CENIC Ciencias Biológicas. 40(1):35-44.

37. VERA, P.; ROJAS, A.; CHÁVEZ, Y.; ARRIAZA, T. 2016. Evaluación de materiales filtrantes para el reúso en agricultura de aguas residuales tratadas provenientes de zonas áridas. Ciencia e Ingeniería Neogranadina. 26(1):5-19. https://doi.org/10.18359/rcin.1633

38. VERA, R.; HOYOS, P. 2019. Long-term beef production from pastures established with and without annual crops compared with native savanna in the Eastern Plains of Colombia: A compilation and analysis of on-farm results 1979-2016. Tropical Grasslands-Forrajes Tropicales. 7(1):113. https://dx.doi.org/10.17138/tgft(7)1-13

39. WANG, S.; DU, J.; ZHAO, L.; JIAO, L.; LI, Z.; XUE, Z. 2019. Tensile and shearing strength properties of the stems of King Grass (Pennisetum sinese Roxb). Chinese J. Tropical Crops. 6:1188-1194.

40. WU, T.; CHANG, X. 2018. Dynamic analysis of rotary cutting system of straw pulverizer. Southern farm machinery. 7:2930. 